\title{
ON THE GEOMETRY OF THE CIRCLE.
}

BY DR. VIRGIL SNYDER:

(Read before the American Mathematical Society, December 28, 1899.)

LET $x_{1}, x_{2}, \cdots, x_{5}$ be any five numbers which satisfy the homogeneous quadratic identity $\omega(x) \equiv 0$; these numbers may be taken as the homogeneous coördinates of the circle.

The linear geometry of the circle can be readily interpreted from the paper of the author* on Dupin's cyclides ; and the corresponding theorems for the quadratic configurations from another paper.t In the latter, one of the numbers $x_{6}$ was given a restricted interpretation, that of representing all the points of space; and the resulting theorems all referred to cyclides. A set of similar theorems exists for the bicircular quartic curves when, in the geometry of the circle, one coördinate equated to zero represents the points of a plane. All the known theorems regarding these curves, as given by Casey, $\$$ Darboux, $\S$ and Loria, $\|$ can be very easily derived, and a number of new ones which are not contained in these memoirs. Another specialization is that obtained by taking the lines of a plane as one of the fundamental complexes. This case has not been systematically treated.

Now suppose that any five complexes mutually in involution be taken as fundamental complexes. In general, none of these complexes is orthogonal, and no circle belongs to them all. The quadratic identity now becomes

$$
\omega(x) \equiv \sum_{\tau=1}^{5} x_{\tau}{ }^{2}=0
$$

These coördinates now represent circles, and not points, as in the memoirs quoted. This theorem results immediately : The curve of singularities of a general quadratic complex is

* V. Snyder, "On the determination of nodes in Dupin's cyclides," Ann. of Math., vol. 11, p. 137.

$\dagger \mathrm{V}$. Snyder, "Geometry of some differential expressions," BuLLETIN, vol. 4 , p. 144 .

$\ddagger$ J. Casey, "On the bicircular quartics," Trans. R. Irish Acad., vol. 24, p. 359.

\%G. Darboux, "Sur une classe remarquable de courbes et de surfaces," Paris, 1873.

$\|$ G. Loria, "Sur la géométrie analytique du cercle," Quar. Jour., of Math., vol. 22, p. 44. 
likewise curve of singularities of $\infty^{1}$ such complexes and is focal curve (or envelope) for five quadratic congruences.

The congruences whose envelopes coincide with the curve of singularities of $\sum_{\tau=1}^{5} a_{\tau} x_{\tau}^{2}=0$ are

$$
x_{\kappa}=0, \quad \sum_{\tau=1}^{5} \frac{x_{\tau}^{2}}{a_{\tau}-a_{\kappa}}=0 \quad(\tau \neq x) .
$$

The locus of centers of circles belonging to any congruence is easily found to be a bicircular quartic, hence :

The curve of singularities of a general quadratic complex is the envelope of circles which cut a fixed circle at a constant angle and whose centers lie on a bicircular quartic.

This curve can be generated in five-fold manner, and if $\varphi_{\tau}, \varphi_{\kappa}$ denote the angles of the $\tau$ th and $x$ th fundamental complexes, and $[\tau, x]$ the angle between their defining circles, then

$$
\cos [\tau, x]=\cos \varphi_{\tau} \cdot \cos \varphi_{\kappa}
$$

for all values of $\tau, x$ from 1 to 5.*

If any coördinate complex is orthogonal, its defining circle belongs to all the other coördinate complexes; if four of the coördinate complexes are orthogonal, the locus of centers in each congruence is a conic section and this case reduces to the one treated before.

By employing the method given on p. 150 of volume 4 of the Bulletin, one easily obtains this theorem :

Eight tangents can be drawn to the curve of singularities parallel to any given line.

One cannot conclude that the curve is of class 8 ; for some particular cases arise in which the line at infinity is a multiple factor in the degraded curve, yet eight parallel tangents can be drawn to the remaining curve in any direction.

The order of the curve is 24 , as may be found as follows : The problem is to find the envelope of the circle

$$
(\mu-\nu)\left(x^{2}+y^{2}\right)-2 \xi x t-2 \eta y t+(\mu+\nu) t^{2}=0,
$$

subject to the three conditions

$$
\begin{gathered}
\omega(\xi, \eta, \lambda, \mu, \nu)=0, \quad F_{2}(\xi, \eta, \lambda, \mu, \nu)=0 \\
a \xi+b \eta+d \lambda+c \mu+f \nu=0
\end{gathered}
$$

\footnotetext{
* See V. Snyder, "Ueber die linearen Complexe der Lie'schen Kugelgeometrie," Göttingen, 1895, p. 37.
} 
or, in terms of the new coördinates, to find the envelope of $\sum_{\tau=1}^{5} s_{\tau} x_{\tau}=0$, subject to the conditions

$$
\sum_{\tau=1}^{5} a_{\tau} x_{\tau}^{2}=0, \quad \sum_{\tau=1}^{5} x_{\tau}^{2}=0, \quad \text { and (say) } \quad x_{1}=0 .
$$

Putting $x_{1}=0$ in all the forms, and differentiating, regarding $x_{5}$ (say) as constant, we obtain

$$
\Delta=\left|\begin{array}{ccc}
s_{2} & s_{3} & s_{4} \\
a_{2} x_{2} & a_{3} x_{3} & a_{4} x_{4} \\
x_{2} & x_{3} & x_{4}
\end{array}\right|=0 .
$$

It now remains to eliminate $x_{2}, x_{3}, x_{4}, x_{5}$ between the four homogeneous equations

$$
\sum_{\tau=2}^{5} s_{\tau} x_{\tau}=0,(1,1) ; \sum x_{\tau}{ }^{2}=0,(2,0) ; \sum a x_{\tau}{ }^{2}=0,(2,0) ; \Delta=0,(2,1) \text {. }
$$

In each parenthesis, the first number denotes the degree in which $x_{\tau}$ enters the equation, and the second the degree in $s_{\tau}^{*}$. Now by the theory of elimination, the $s_{\tau}$ from the first equation will enter the $x$ eliminant to the degree 2.2.2, and those from $\Delta$ to the degree 2.2.1 ; hence the $s_{\tau}$ appear to degree 12. But $s_{\tau}=0$ is the cartesian equation of a circle, hence the curve of singularities is of degree 24 .

The curve passes 12 times through each circle point; its equation contains 12 constants.

Straight lines, conics, circular cubics, and bicircular quartics, as well as their parallels, evolutes, and the involutes of their caustics by reflection, can be exhaustively classified from this standpoint; they all appear as degraded forms of the curve of singularities.

The lines drawn perpendicular to the asymptotes of the bicircular quartic (locus of centers), and cutting the fundamental circle of $x_{\tau}$ at. the angle of the complex $\varphi_{\tau}$, are all bitangents to the curve of singularities. The bicircular quartic has 4 asymptotes and there are two double tangents associated with each ; hence each fundamental circle gives rise to 8 double tangents, all of which are tangent to a circle concentric with the given one. There are five such sets or forty in all (besides the line at infinity which may be a multiple tangent). The asymptotes of bicircular quartics, however, are all isotropic and perpendiculars to them must coincide with the lines themselves. They therefore fall to- 
gether in pairs and pass through the centers of the five fundamental circles; hence :

The bitangents of the curve of singularities are all isotropic; one pair passes through the center of each fundamental circle.

The four points in which each circle intersects its associated quartic are foci of the curve of singularities; hence the latter has 20 single foci. There are 12 double foci, but they may not all be distinct.

By varying $c$ in the equations

$$
x_{k}=0, \quad \sum_{\tau=1}^{5} \frac{x_{\tau}^{2}}{a_{\tau}-c}=0
$$

a family of cosingular congruences is obtained, having a series of focal curves all of which pass through a finite number of fixed points. These curves are distributed into five distinct families. Two curves of the system pass through every point in the plane, the angle of intersection being a determinate function of $\varphi_{\tau}, \varphi_{\kappa}$ obtained by considering the tangent congruences of the complexes defining the curve at the given point. Each of the five sets contains a pair of circles as a degraded focal curve.

In a similar manner the following theorem may be proved :

The envelope of circles which cut a fixed circle orthogonally and whose centers lie on a curve of class $n$ is a curve of order $2 n$, which. passes $n$ times through each circle point.

Its foci and double tangents are easily determined as before.

A corresponding generalization can be made in the geometry of the sphere. The depiction of the Kummer surface in general sphere space is the envelope of spheres whose centers lie on a cyclide and which cut a fixed sphere at a constant angle. The same surface can be generated in six ways, though the loci of centers are not in general confocal nor are the fixed spheres orthogonal. Twelve tangent planes can be drawn parallel to any given plane. The cones of Kummer of bitangent planes which belong to the cyclide give rise to six developables of tangent planes to the imaginary circle at infinity. The lines of curvature on these developables are defined by pencils of spheres concentric with the fundamental spheres.*

This surface appears to be of order 64, contains the circle at infinity as a 32-fold line, and depends on 18 constants.

CORNELL UNIVERSITY, December 28, 1899.

* See V. Snyder. "Lines of curvature on annular surfaces." Amer. Jour. of Math., vol. 22, p. 96. 